\title{
CANTOR-TYPE UNIQUENESS OF MULTIPLE TRIGONOMETRIC INTEGRALS
}

\author{
VICTOR L. SHAPIRO
}

1. Introduction. It is the purpose of this paper to obtain results in Cantortype uniqueness for multiple trigonometric integrals similar to those obtained previously for multiple trigonometric series $([\mathbf{5}, \mathbf{1 1}, \mathbf{1 2}])$. As might be expected, the results in the integral case are a bit more difficult to obtain.

Vectorial notation is used for the most part throughout this paper. Thus $u$ designates the point in $n$-dimensional euclidean space, $E_{n}$, with coordinates $\left(u_{1}, \cdots, u_{n}\right)$, the scalar product $(u, x)=u_{1} x_{1}+\cdots+u_{n} x_{n}$, with $|u|=(u, u)^{1 / 2}$ and $u+\alpha x$ is the point $\left(u_{1}+\alpha x_{1}, \cdots, u_{n}+\alpha x_{n}\right)$.

Previously the author [13], using equisummability between trigonometric integrals and trigonometric series, has obtained in the special case of double trigonometric integrals the following result:

Let $c(u)$, in $L_{2}$ on any bounded domain, be $O\left(|u|^{-\epsilon}\right), \epsilon>0$. Suppose the double trigonometric integral $\int_{E_{2}} e^{i(x, u)} c(u) d u$ is circularly summable $(C, 1)$ to $f(x)$. Furthermore suppose $f(x)$ is in Lip $\alpha, \alpha>0$, on every bounded domain ( $\alpha$ depending on the domain). Then the double trigonometric integral

$$
\frac{1}{4 \pi^{2}} \int_{E_{2}} e^{-i(x, u)} f(x) d x
$$

is spherically summable $(C, 1)$ to $c(u)$ for almost every $u$.

Specializing $f(x)$ to be the zero function (which is what is meant by Cantortype uniqueness, $[15, \mathrm{p} .274]$ ) and using a more direct attack on the problem, we are able in this paper both to weaken the hypotheses of the above theorem as well as to extend the results to $n$-dimensional integrals.

2. Definitions and notation. The open $n$-dimensional sphere with center $x$ and radius $r$ will be designated by $D_{n}(x, r)$, and the surface of the sphere by $C_{n}(x, r)$.

Received November 26, 1953. Presented to the American Mathematical Society November 27, 1953. This investigation was supported in part by a grant from the Rutgers University Research Fund.

Pacific J. Math. 5 (1955), 607-622 
Following Bochner [1], we shall say that the multiple trigonometric integral $\int_{E_{n}} c(u) e^{i(x, u)} d u$ is spherically convergent at the point $x$ to the finite value $L(x)$ if the spherical partial integrals of rank $R$ converge to $L(x)$, that is if

$$
I_{R}(x)=\int_{D_{n}(0, R)} e^{i(x, u)} c(u) d u \rightarrow L(x) \quad(\text { as } R \rightarrow \infty .)
$$

The integral

$$
\sigma_{R}^{(\alpha)}(x)=2 \alpha R^{-2 \alpha} \int_{0}^{R} I_{r}(x)\left(R^{2}-r^{2}\right)^{\alpha-1} r d r, \alpha>0,
$$

is called the $(C, \alpha)$-mean of rank $R$ of the multiple trigonometric integral $\int_{E_{n}} c(u) e^{i(x, u)} d u$, and this integral is said to be spherically summable $(C, \alpha)$ to $L(x)$ if $\sigma_{R}^{(\alpha)}(x) \longrightarrow L(x)$ as $R \rightarrow \infty$.

Given $F(x)$ integrable on $D_{n}\left(x_{0}, r\right)$, we designate the mean value of $F$ in this sphere by $A\left(F ; x_{0} ; r\right)$. Given $F(x)$ integrable on $C_{n}\left(x_{0} ; r\right)$, we designate the mean value of $F$ on this surface by $L\left(F ; x_{0} ; t\right)$. Thus, designating the volume of the unit $n$-dimensional sphere, $2 \pi^{n / 2} / n \Gamma(n / 2)$, by $\Omega_{n}$ and the $(n-1)$ dimensional volume of its surface, $2 \pi^{n / 2} / \Gamma(n / 2)$, by $\omega_{n}$, we have

$$
\begin{aligned}
& A\left(F ; x_{0} ; r\right)=\left(\Omega_{n} r^{n}\right)^{-1} \int_{D_{n}\left(x_{0}, r\right)} F(x) d x \\
& L\left(F ; x_{0} ; r\right)=\omega_{n}^{-1} \int_{C_{n}(0,1)} F\left(x_{0}+r x\right) d S_{n-1}(x)
\end{aligned}
$$

where $d S_{n-1}$ is the $(n-1)$ dimensional volume element of $C_{n}(0,1)$.

We set

$$
\nabla_{1}\left(F ; x_{0} ; r\right)=L\left(F ; x_{0} ; r\right)-F\left(x_{0}\right) \text { and } \nabla_{2}\left(F ; x_{0} ; r\right)=A\left(F ; x_{0} ; r\right)-F\left(x_{0}\right)
$$

and say that $F(x)$ has a generalized Laplacian of the first or second kind at the point $x_{0}$ equal to $\alpha_{1}$ or $\alpha_{2}$, respectively, if

$$
\lim _{r \rightarrow 0} 2 n \nabla_{1}\left(F ; x_{0} ; r\right) / r^{2}=\alpha_{1}
$$

or

$$
\lim _{r \rightarrow 0} 2(n+2) \nabla_{2}\left(F ; x_{0} ; r\right) / r^{2}=\alpha_{2} \text {. }
$$


The generalized Laplacian of the first and second kind of $F$ at $x_{0}$ will be designated by $\Delta_{1} F\left(x_{0}\right)$ and $\Delta_{2} F\left(x_{0}\right)$, respectively. It is known, [6, p. 261], that if $F(x)$ is in class $C^{(2)}$ on $D_{n}\left(x_{0}, r_{0}\right)$, then $\Delta F\left(x_{0}\right)=\Delta_{1} F\left(x_{0}\right)=\Delta_{2} F\left(x_{0}\right)$ where $\Delta F(x)$ is the ordinary Laplacian of $F$ at $x$.

The closure of the set $\mathbb{W}$ is designated by $\bar{W}$; and its characteristic function by $\chi_{\mathbb{W}}(x)$. The set $Z$ is said to be a closed set of vanishing capacity if for every $r, Z \bar{D}_{n}(0, r)$ is a closed set of capacity zero. It is known, [4], that if $Z$ is a set of vanishing capacity then $D_{n}\left(x_{0}, r\right)-Z D_{n}\left(x_{0}, r\right)$ is a domain.

The trigonometric integral $\int_{E_{n}} e^{i(x, u)} c(u) d u$ is said to be of type $(U)$ on a domain $G$ if

$$
\int_{E_{n}-D_{n}(0,1)} e^{i(x, u)} c(u)|u|^{-2} d u
$$

converges spherically on $G$ to a function $F(x)$ which is continuous on $G$.

Throughout this paper $E_{n}$ stands for $n$-dimensional euclidean space where $n \geq 2$, and $\mu=(n-2) / 2$.

The function $J_{i}(r)$ is the Bessel function of the first kind of order $i$.

3. Statement of main results. We shall prove the following two theorems concerning Cantor-type uniqueness for multiple trigonometric integrals.

THEOREM 1. Given the multiple trigonometric integral $\int_{E_{n}} e^{i(x, u)}{ }_{c}(u) d u$ where $c(u)$ is a complex-valued function which is integrable on every bounded domain. Let $Z$ be a closed set of vanishing capacity. Suppose that

(i) The integral is spherically summable $(C, 1)$ to zero almost everywhere.

(ii) The $(C, 1)$ spherical mean of rank $R, \sigma_{R}^{(1)}(x)$, is such that $\overline{\lim }_{R \rightarrow \infty}$ $\left|\sigma_{R}^{(1)}(x)\right|<\infty$ in $E_{n}-Z$.

(iii) $c(u)\left(|u|^{2}+1\right)^{-1}$ is in $L_{1}$ on $E_{n}$.

Then $c(u)$ vanishes almost everywhere.

THЕовем 2. Given the multiple trigonometric integral $\int_{E_{n}} e^{i(x, u)}{ }_{c}(u) d u$ where $c(u)$ is a complex-valued function which is integrable on every bounded domain. Let $Z$ be a closed set of vanishing capacity. Suppose that

(i) and (ii) The same as (i) and (ii) of Theorem 1.

(iii) The integral is of type $(U)$ on $E_{n}$.

(iv) $c(u)\left(|u|^{2}+1\right)^{-1}$ is in $L_{2}$ on $E_{n}$.

Then $c(u)$ vanishes almost everywhere. 
For the special case of the plane, we prove the following theorem.

THEOREM 3. Given the double trigonometric integral $\int_{E_{n}} e^{i(x, u)} c(u) d u$ where $c(u)$ is a complex-valued function which is integrable on every bounded domain. Let $Z$ be a closed set of vanishing capacity and $\mathbb{W}$ be a closed denumerable set such that $W Z=0$. Suppose that

(i) The integral is spherically summable $(C, 1)$ to zero in $E_{2}-Z$.

(ii) The integral is of type $(U)$ on $E_{2}-W$.

(iii) $c(u)=o(|u|)$ as $|u| \rightarrow \infty$

(iv) $c(u)\left(|u|^{2}+1\right)^{-1}$ is in $L_{2}$ on $E_{n}$.

Then $c(u)$ vanishes almost everywhere.

4. Fundamental lemmas. Before proving the main theorems of this paper, it is first necessary to establish a connection between the $(C, 1)$ spherical summability of the integral $\int_{E_{n}} e^{i(x, u)} c(u) d u$ and the generalized Laplacians of the "anti-Laplacian" of this integral. In short, we shall now establish some Riemann-type, [15, p. 270 ], results for the multiple trigonometric integrals.

We need prove the following lemma only for the plane, since the conclusion is hypothesized for Theorems 1 and 2.

LEMmA 1. Let $c(u)$ be a complex-valued function which is integrable on every bounded domain in the plane, vanishes in $D_{2}\left(0, r_{0}\right), r_{0}>0$, and is $o(|u|)$. Suppose that $\sigma_{R}^{(1)}\left(x_{0}\right)=o(R)$ where $\sigma_{R}^{(1)}(x)$ is the $(C, 1)$ spherical mean of rank $R$ of $\int_{E_{2}} e^{i(x, u)} c(u) d u$. Then $\int_{E_{2}} e^{i(x, u)} c(u)|u|^{-2} d u$ is spherically convergent.

Without loss of generality, we assume $x_{0}$ to be the origin. Then with $I_{R}(x)$ given by (1) and $\sigma_{R}^{(1)}(x)$ by (2), we have

$$
\begin{aligned}
\int_{D_{2}(0, R)} c(u)|u|^{-2} d u & =2 \int_{D_{2}(0, R)} c(u) d u \int_{0}^{R} \chi_{D_{2}(0, r)}(u) r^{-3} d r+R^{-2} I_{R}(0) \\
& =2 \int_{0}^{R} r^{-3} d r \int_{D_{2}(0, R)} c(u) \chi_{D_{2}(0, r)}(u) d u+R^{-2} I_{R}(0) \\
& =2 \int_{0}^{R} r^{-3} I_{r}(0) d r+R^{-2} I_{R}(0) \\
& =4 \int_{0}^{R} \sigma_{r}^{(1)}(0) r^{-3} d r+\sigma_{R}^{(1)}(0) R^{-2}+R^{-2} I_{R}(0) .
\end{aligned}
$$


Since by assumption $\sigma_{R}^{(1)}(0)=o(R)$, to prove the lemma it only remains to show that $I_{R}(0)=o\left(R^{2}\right)$. But

$$
\begin{aligned}
I_{R}(0)=\left[(R+1)^{2} \sigma_{R+1}^{(1)}(0)-R^{2} \sigma_{R}^{(1)}(0)\right](2 R+1)^{-1} \\
\left.\quad-(2 R+1)^{-1} \int_{D_{2}(0, R+1)-D_{2}(0, R)} c(u)[R+1)^{2}-|u|^{2}\right] d u=o\left(R^{2}\right),
\end{aligned}
$$

and the proof is complete.

LEMMA 2. Let $c(u)$ be a complex-valued function which is integrable on every bounded domain in $E_{n}$ and which vanishes in $D_{n}\left(0, r_{0}\right), r_{0}>0$. Suppose that

(i) $\widetilde{\lim }_{R \rightarrow \infty}\left|\sigma_{R}^{(1)}\left(x_{0}\right)\right|=d$ where $\sigma_{R}^{(1)}\left(x_{0}\right)$ is the $(C, 1)$ spherical mean of rank $R$ of $\int_{E_{n}} e^{i(x, u)} c(u) d u$ and $d$ is finite-valued.

$$
\text { (ii) }-\int_{E_{n}} e^{i(x, u)} c(u)|u|^{-2} d u
$$

is spherically uniformly convergent in $\nu_{n}\left(x_{0}, t_{0}\right), t_{0}>0$, to $F(x)$.

Then $\overline{\lim }_{t \rightarrow 0}\left|2 n \nabla_{1}\left(F ; x_{0} ; t\right) / t^{2}\right| \leq K d$ where $K$ is a constant independent of $x_{0}$ and $d$.

Observing that for fixed $u$

$$
L\left(e^{i(x, u)} ; x_{0} ; t\right)=2^{\mu} \Gamma(\mu+1) e^{i\left(u, x_{0}\right)}(|u| t)^{-\mu} J_{\mu}(|u| t)
$$

( $\operatorname{see}[1, p .177]$ ), we have by assumption (ii) for $t$ sufficiently small that

$$
L\left(F ; x_{0} ; t\right)
$$

$$
=-2^{\mu} \Gamma(\mu+1) \lim _{R \rightarrow \infty} \int_{D_{n}(0, R)} e^{i\left(x_{0}, u\right)} c(u)|u|^{-2} J_{\mu}(|u| t)(|u| t)^{-\mu} d u
$$

and consequently that

$$
(2 n) \nabla_{1}\left(F ; x_{0} ; t\right) / t^{2}=\lim _{R \rightarrow \infty} \int_{D_{n}(0, R)} e^{i\left(x_{0}, u\right)} c(u) \eta(|u| t) d u
$$

where

$$
\eta(r)=4(\mu+1)\left[1-2^{\mu} \Gamma(\mu+1) J_{\mu}(r) r^{-\mu}\right] / r^{2} \text { for } r>0, \eta(0)=1
$$


and $\eta(r)$ is in $C^{(\infty)}$.

Making the following observations:

(a) By the second mean-value theorem applied to the real and imaginary parts of $I_{R}\left(x_{0}\right)$ given by $(1)$ and hypothesis (ii) we have $I_{R}\left(x_{0}\right)=o\left(R^{2}\right)$,

(b) For fixed $t, \eta(R t)=O\left(R^{-2}\right)$ and $\eta^{\prime}(R t)=O\left(R^{-5 / 2}\right)$ where $\eta^{\prime}(r)=$ $d \eta(r) / d r$, we obtain from (4) that

$$
2 n \nabla_{1}\left(F ; x_{0} ; t\right) / t^{2}=2^{-1} \int_{0}^{\infty} r^{2} \sigma_{r}^{(1)}\left(x_{0}\right) t^{3} a(r t) d r
$$

where $\alpha(r)=d r^{-1} \eta^{\prime}(r) / d r$.

From the fact that $a(z)$ is an entire function of the form $\sum_{i=0}^{\infty} b_{i} z^{2 i+1}$, we have that there exists a constant $K_{1}$ such that

$$
|a(r)| \leq K_{1} r \text { for } r \leq 1
$$

From the fact that $J_{\mu}(r)=O\left(r^{-1 / 2}\right)$ as $r \longrightarrow \infty$, and

$$
d r^{-\mu} J_{\mu}(r) / d r=-r^{-\mu} J_{\mu+1}(r),
$$

we obtain that there exists a constant $K_{2}$ such that

$$
\left|c_{2}(r)\right| \leq K_{2}\left[r^{-(\mu+7 / 2)}+r^{-5}\right] \quad \text { for } r \geq 1
$$

From (5), (6), and (7), the conclusion of the lemma follows readily. For given an $\epsilon>0$, choose $R_{0}$ so large that $\left|\sigma_{R}^{(1)}\left(x_{0}\right)\right| \leq d+\epsilon$ for $R>R_{0}$. Then for $t<R_{0}^{-1}$, it is easily seen that

$$
\left|2 n \nabla_{1}\left(F ; x_{0}, t\right) / t^{2}\right| \leq K(d+\epsilon)+O\left(t^{4}\right)
$$

where $K$ is a constant independent of $x_{0}, d$, and $\epsilon$. Taking the limit superior of the left side of ( 8$)$ as $t \rightarrow 0$ and then the limit of the right side as $\epsilon \rightarrow 0$, we have the proof of the lemma.

Lemma 3. Let the hypotheses be the same as in Lemma 2 except that $\lim _{\mathrm{R} \rightarrow \infty} \sigma_{R}^{(1)}\left(x_{0}\right)=d$. Then $\Delta_{1} F\left(x_{0}\right)=d$.

For if $d=0$, the lemma follows immediately from Lemma 2. If $d \neq 0$, choose $c_{1}(u)$ integrable on $E_{n}$, vanishing for $u$ in $\left[E_{n}-D_{n}(0,2)\right]+D_{n}(0,1)$, and such that $\int_{E_{n}} c_{1}(u) e^{i\left(x_{0}, u\right)}=d$. Set $F_{1}(x)=-\int_{E_{n}} c_{1}(u)|u|^{-2} e^{i(x, u)} d u$. Then 
$0=\Delta_{1} F\left(x_{0}\right)-\Delta_{1} F_{1}\left(x_{0}\right)=\Delta_{1} F\left(x_{0}\right)-\Delta F_{1}\left(x_{0}\right)=\Delta_{1} F\left(x_{0}\right)-d$.

LEMMA 4. Let $c(u)$ be a complex valued function which is integrable on every bounded domain in $E_{n}$ and which vanishes in $D_{n}\left(0, r_{0}\right), r_{0}>0$. Sup pose that

(i) $\sigma_{R}^{(1)}(x)$, the $(C, 1)$ spherical mean of rank $R$ of $\int_{E_{n}} e^{i(x, u)} c(u) d u$, is such that $\overline{\lim }_{R \rightarrow \infty}\left|\sigma_{R}^{(1)}\left(x_{0}\right)\right|=d$.

(ii) $c(u)|u|^{-2}$ is in $L_{2}$ on $E_{n}$.

$$
\text { (iii) }-\int_{E_{n}} e^{i\left(x_{0}, u\right)}|u|^{-2} c(u) d u
$$

is spherically convergent to $F\left(x_{0}\right)$. Set

$$
F(x)=\underset{R \rightarrow \infty}{\operatorname{lim.m.}} \int_{D_{n}(0, R)} e^{i(x, u)} c(u)|u|^{-2} d u .
$$

Then

$$
\varlimsup_{t \rightarrow 0}\left|2(n+1) \nabla_{2}\left(F ; x_{0} ; t\right) / t^{2}\right| \leq K d
$$

where $K$ is a constant independent of $x_{0}$ and $d$.

\section{Setting}

$$
T_{R}(x)=-\int_{D_{n}(0, R)} e^{i(x, u)} c(u)|u|^{-2} d u
$$

observing that $A\left(F ; x_{0} ; t\right)=\lim _{R \rightarrow \infty} A\left(T_{R} ; x_{0} ; t\right)$ and that for fixed $u$,

$$
\begin{aligned}
A\left(e^{i(x, u)} ; x_{0} ; t\right) & =\omega_{n} / \Omega_{n} t^{n} \int_{0}^{t} r^{n-1} L\left(e^{i(x, u)} ; x_{0} ; r\right) d r \\
& =2^{\mu+1} \Gamma(\mu+2)(|u| t)^{-(\mu+1)} J_{\mu+1}(|u| t) e^{i\left(x_{0}, u\right)},
\end{aligned}
$$

we obtain

(9) $A\left(F ; x_{0} ; t\right)$

$$
=-\lim _{R \rightarrow \infty} 2^{\mu+1} \Gamma(\mu+2) \int_{D_{n}(0, R)} e^{i\left(x_{0}, u\right)} c(u)|u|^{-2}(j u \mid t)^{-(\mu+1)} J_{\mu+1}(|u| t) d u
$$

and consequently 


$$
2(n+2) \nabla_{2}\left(F ; x_{0} ; t\right) / t^{2}=\lim _{R \rightarrow \infty} \int_{D_{n}(0, R)} e^{i\left(x_{0}, u\right)} \gamma(|u| t) c(u) d u
$$

where

$$
\gamma(r)=4(\mu+2)\left[1-2^{(\mu+1)} \Gamma(\mu+2) r^{-(\mu+1)} J_{\mu+1}(r)\right] / r^{2} \text { for } r>0, \gamma(0)=1 \text {, }
$$

and $\gamma(r)$ is in $C^{(\infty)}$.

Since $\gamma(r)$ has the same form as $\eta(r)$ in Lemma 2 with $\mu$ replaced by $\mu+1$, we can proceed as in that lemma and obtain

$$
2(n+2) \nabla_{2}\left(F ; x_{0} ; t\right) / t^{2}=2^{-1} \int_{0}^{\infty} t^{3} r^{2} \sigma_{r}^{(1)}\left(x_{0}\right) \beta(r t) d r
$$

where $\beta(r)=d r^{-1} \gamma^{\prime}(r) / d r$. Then we can proceed in a similar manner to obtain that for $\epsilon>0$

$$
\varlimsup_{t \rightarrow \infty}\left|2(n+2) \nabla_{2}\left(F ; x_{0} ; t\right) / t^{2}\right| \leq K(d+\epsilon)
$$

where $K$ is a constant independent of $x_{0}, d$, and $\epsilon$. Since $\epsilon$ is arbitrary the conclusion of the lemma follows.

LEMMA 5. Let the hypotheses be the same as in Lemma 4 except that $\lim _{R \rightarrow \infty} \sigma_{R}^{(1)}\left(x_{0}\right)=d$. Then $\Delta_{2} F\left(x_{0}\right)=d$.

In the same manner that we obtained Lemma 3 from Lemma 2, we obtain Lemma 5 from Lemma 4.

Lemma 6. Let $F(x)$ be real-valued and continuous on $\bar{D}_{n}\left(x_{0}, r_{0}\right), r_{0}>0$. Suppose that

(i) $\Delta_{2} F(x)=0$ almost everywhere in $D_{n}\left(x_{0}, r_{0}\right)$

(ii) $\varlimsup_{r \rightarrow \infty}\left|2(n+2) \nabla_{2}(F ; x ; r) / r^{2}\right|<\infty$ for all $x$ in $D_{n}\left(x_{0}, r_{0}\right)$.

Then $F(x)$ is harmonic in $D_{n}\left(x_{0}, r_{0}\right)$.

Following the pattern of proof in [9], we give a proof for $n \geq 3$.

To prove the lemma, it is sufficient to show that $F(x)$ is subharmonic in $D_{n}\left(x_{0}, r_{0}\right)$.

Set 


$$
f(x)=2(n+2)\left[\varlimsup_{r \rightarrow 0} \nabla_{2}(F ; x ; r) / r^{2}+\underset{r \rightarrow 0}{\lim } \nabla_{2}(F ; x ; r) / r^{2}\right] / 2
$$

for $x$ in $D_{n}\left(x_{0}, r_{0}\right)$. Then $f(x)=0$ almost everywhere in $L_{n}\left(x_{0} ; r_{0}\right)$.

By the theorem of Vitali-Caratheodory $[10$, p. 75], there exists a sequence of nondecreasing upper semicontinuous functions $\left\{g_{m}(x)\right\}$ such that $g_{m}(x) \leq$ $f(x)$ for all $x$ in $D_{n}\left(x_{0}, r_{0}\right), g_{m}(x) \rightarrow f(x)$ almost everywhere in $D_{n}\left(x_{0}, r_{0}\right)$, $g_{m}(x)$ is integrable on $\nu_{n}\left(x_{0}, r_{0}\right)$, and such that

$$
\lim _{m \rightarrow \infty} \int_{D_{n}\left(x_{0}, r\right)} g_{m}(x) d x=\int_{D_{n}\left(x_{0}, r\right)} f(x) d x \quad \text { for } r \leq r_{0} .
$$

Set

$$
\Delta^{-1} g_{m}(x)=-\left[\omega_{n}(n-2)\right]^{-1} \int_{D_{n}\left(x_{0}, r_{0}\right)} g_{m}(u)|u-x|^{2-n} d u .
$$

Then $\Delta^{-1} g_{m}(x)$ is superharmonic, since $g_{m}(u) \leq 0$ for almost all $u$ in $D_{n}\left(x_{0}, r_{0}\right)$. Furthermore, we observe that for fixed $u$

$$
\begin{aligned}
A\left(|x-u|^{2-n} ; x_{0} ; r\right) & =\left|x_{0}-u\right|^{2 n} \text { if }\left|x_{0}-u\right|>r \\
& =n r^{-n} 2^{-1}\left[r^{2}+\left|x_{0}-u\right|^{2}(2-n) n^{-1}\right] \text { if }\left|x_{0}-u\right| \leq r .
\end{aligned}
$$

Consequently, for $x_{1}$ in $D_{n}\left(x_{0}, r\right)$ with $r$ sufficiently small,

$$
\begin{aligned}
\nabla_{2}\left(\Delta^{-1} g_{m} ; x_{1}, r\right)= & {\left[\omega_{n}(n-2)\right]^{-1} \int_{D_{n}\left(x_{1}, r\right)} g_{m}(u)\left\{\left|u-x_{1}\right|^{2-n}\right.} \\
& \left.-n r^{-n} 2^{-1}\left[r^{2}+\left|x_{1}-u\right|^{2}(2-n) n^{-1}\right]\right\} d u .
\end{aligned}
$$

Suppose $g_{m}\left(x_{1}\right)$ is finite. Then by the upper semi-continuity of $g_{m}(u)$ at $x_{1}$, for $\epsilon>0$ and $r$ sufficiently small, we have from (11) that

$$
\nabla_{2}\left(\Delta^{-1} g_{m} ; x_{1} ; r\right) \leq\left[g_{m}\left(x_{1}\right)+\epsilon\right]\left[\omega_{n}(n-2)\right]^{-1}\left[\omega_{n}(n-2)\right] r^{2} / 2(n+2) .
$$

Consequently, we conclude that

$$
\varlimsup_{r \rightarrow 0} 2(n+2) \nabla_{2}\left(\Delta^{-1} g_{m} ; x_{1} ; r\right) / r^{2} \leq g_{m}\left(x_{1}\right)
$$

Similarly, in case $g_{m}\left(x_{1}\right)=-\infty$, conclusion (12) remains valid. 
From the fact that $\Delta^{-1} g_{m}(x)$ is superharmonic, we have that $F-\Delta^{-1} g_{m}$ is upper semi-continuous in $D_{n}\left(x_{0}, r_{0}\right)$. From (12) we conclude that

$$
\varlimsup_{r \rightarrow 0} 2(n+2) \nabla_{2}\left(F-\Delta^{-1} g_{m} ; x ; r\right) / r^{2} \geq 0 \quad \text { for } x \text { in } D_{n}\left(x_{0}, r_{0}\right) .
$$

Therefore by $[8$, p. 14$],\left\{F-\Delta^{-1} g_{m}\right\}$ is a nondecreasing sequence of subharmonic functions in $D_{n}\left(x_{0}, r_{0}\right)$. But $\lim _{m \rightarrow \infty} \Delta^{-1} g_{m}(x)=0$ almost everywhere. Therefore $F(x)$ is almost everywhere equal to a subharmonic function, $G(x)$, by $\left[8\right.$, p. 22]. But $A(F ; x ; r)=A(G ; x ; r) \rightarrow G(x)$ for all $x$ in $D_{n}\left(x_{0}, r_{0}\right)$. However from the continuity of $F$ we have $A(F ; x ; r) \rightarrow F(x)$, and the proof of the lemma is complete for $n \geq 3$. For $n=2$ a similar proof can be given with the Newtonian potential replaced by the logarithmic potential.

For the case of the generalized Laplacian of the first kind, we have a similar lemma with a similar proof, see [9].

LEMMA 7. Let $F(x)$ be real-valued and continuous on $\bar{D}_{n}\left(x_{0}, r_{0}\right), r_{0}>0$. Suppose that

(i) $\Delta_{1} F(x)=0$ almost everywhere in $D_{n}\left(x_{0}, r_{0}\right)$.

(ii) $\varlimsup_{r \rightarrow 0}\left|2 n \nabla_{1}(F ; x ; r) / r^{2}\right|<\infty$ for all $x$ in $D_{n}\left(x_{0} ; r_{0}\right)$.

Then $F(x)$ is harmonic in $D_{n}\left(x_{0}, r_{0}\right)$.

We now prove some lemmas concerning the spherical summability $(C, n)$ of Fourier transforms.

Lemma 8. Let $G(x)$ be a function in $L_{1}$ on $E_{n}$ which vanishes in $D_{n}\left(0, r_{0}\right)$, $r_{0}>0$. Suppose that $F(x)=\int_{E_{n}} e^{i(x, u)} G(u) d u$ is in $C^{(2)}$ on $E_{n}$. Then for $u$ in $D_{n}\left(o, r_{0} / 2\right)-0$

$$
\int_{E_{n}}\left[e^{-i(x, u)} F(x)-\left(-e^{-i(x, u)}|u|^{-2} \Delta F(x)\right)\right] d x
$$

is spherically summable $(C, n)$ to zero.

For, by Green's second identity, we have

$$
\begin{aligned}
I_{R}(u) & =\int_{D_{n}(0, R)}\left[e^{-i(x, u)} F(x)-\left(-e^{-i(x, u)}|u|^{-2} \Delta F(x)\right)\right] d x \\
& =|u|^{-2}\left\{R^{n-1} \int_{C_{n}(0,1)} F(R x) i(x, u) e^{-i R(x, u)} d S_{n-1}(x)\right.
\end{aligned}
$$




$$
\left.+R^{n-1} \int_{C_{n}(0,1)} \partial F(R x) / \partial R e^{-i R(x, u)} d S_{n-1}(x)\right\} \equiv|u|^{-2}\left(A_{R}+B_{R}\right)
$$

where $d S_{n-1}(x)$ is the $(n-1)$ dimensional volume element on the unit sphere $C_{n}(0,1)$.

We shall now show that both $A_{R}$ and $B_{R}$ are $(C, n)$ summable to zero. For, by Fubini's theorem, we have

$$
\begin{aligned}
& \left(M R^{2}\right)^{-1} \int_{0}^{\infty} r \phi_{n}\left(\frac{r}{R}\right) A_{r} d r \\
& \quad=\left(M R^{2}\right)^{-1} i \int_{E_{n}-D_{n}\left(0, r_{0}\right)} G(y)\left[\int_{E_{n}} \dot{\phi}_{n}\left(\frac{|x|}{R}\right) e^{-i(x, u-y)}(x, u) d x\right] d y
\end{aligned}
$$

where $M=(2 \pi)^{n / 2} / 2^{n-1}(n-1) !$ and

$$
\phi_{1}(r)=\left\{\begin{array}{ll}
1 & \text { for } 0 \leq r \leq 1 \\
0 & \text { for } r>1
\end{array} \quad \phi_{n}(r)=\left\{\begin{array}{ll}
\left(1-r^{2}\right)^{n-1} & \text { for } 0 \leq r \leq 1 \\
0 & \text { for } r>1
\end{array} .\right.\right.
$$

Since for fixed $u \neq 0,(x, u)$ is a homogeneous polynomial which is also a harmonic function in $x$, we have by $[2, \mathrm{p} .806]$ and $[14, \mathrm{p} .373]$ that the right side of $(15)$ is equal to

$$
-R^{-1} \int_{E_{n}-D_{n}\left(0, r_{0}\right)} G(y) \frac{(y-u, u)}{|y-u|^{n+1}} \frac{J_{n+\mu+1}(R|y-u|) d y}{(R|y-u|)^{n-\mu-2}} .
$$

Clearly (16) tends to zero as $R \longrightarrow \infty$; so $A_{R}$ is $(C, n)$ summable to zero for $u$ in $D_{n}\left(0, r_{0} / 2\right)-0$.

We also observe after integrating by parts that

$$
\begin{aligned}
& \left(M R^{2}\right)^{-1} \int_{0}^{R} r \phi_{n}\left(\frac{r}{R}\right) B_{r} d r \\
& \quad=\left(M R^{2}\right)^{-1} \int_{0}^{R} d r \int_{C_{n}(0,1)} F(r x) \frac{\partial}{\partial r}\left[r^{n} \phi_{n}\left(\frac{r}{R}\right) e^{-i r(x, u)}\right] d S_{n-1}(x) .
\end{aligned}
$$

From the above discussion concerning $A_{R}$ and from [1, Theorem 1], to show that $B_{R}$ is $(C, n)$ summable to zero for $u$ in $D_{n}\left(0, r_{0} / 2\right)-0$, it is sufficient to show that 


$$
\left(M R^{2}\right)^{-1} \int_{E_{n}} F(x) \phi_{n-1}\left(\frac{|x|}{R}\right) \frac{|x|^{2}}{R^{2}} e^{-i(x, u)} d x \rightarrow 0 \text { as } R \rightarrow \infty
$$

But by $[2$, p. 806] and $[14, p .373]$ the expression in (18) is equal to

$$
\begin{aligned}
& R^{-1} \int_{E_{n}-D_{n}\left(0, r_{0}\right)} \frac{G(y)}{|y-u|^{n-1}}\left[K_{1} \frac{J_{n+\mu}(R|y-u|)}{(R|y-u|)^{n-\mu-1}}\right. \\
& \left.\quad-K_{2} \frac{J_{n+\mu-1}(R|y-u|)}{\left(R|y-u|^{n-\mu-2}\right)}\right] d y .
\end{aligned}
$$

where $K_{1}$ and $K_{2}$ are two constants depending on $n$.

Clearly (19) tends to zero as $R \rightarrow \infty$ for $u$ in $D_{n}\left(0, r_{0} / 2\right)-0$; so $B_{R}$ is $(C, n)$ summable to zero and the lemma is proved.

LEMмA 9. Let $G(x)$ be a function in $L_{2}$ on $E_{n}^{\prime}$ which vanishes in $D_{n}\left(0, r_{U}\right)$, $r_{0}>0$. Suppose that $\int_{E_{n}} e^{i(x, u)} G(x) d x$ is spherically convergent to a function $F(x)$ which is in $C^{(2)}$ on $E_{n}$. Then for $u$ in $D_{n}\left(0, r_{0} / 2\right)-0$

$$
\int_{E_{n}}\left[e^{-i(x, u)} F(x)-\left(-e^{-i(x, u)}|u|^{-2}\right) \Delta F(x)\right] d x
$$

is spherically summable $(C, n)$ to zero.

For (14) also holds in this case, and as in Lemma 8, we have to show that both $A_{R}$ and $B_{R}$ are $(C, n)$ summable to zero.

Since both $F(x)$ and $\phi_{n}(|x| / R)(x, u)$ are in $L_{2}$ on $E_{n}$, Parseval's formula gives us both (15) and (16). We therefore conclude as before that $A_{R}$ is $(C, n)$ summable to zero for $u$ in $D_{n}\left(0, r_{0} / 2\right)-0$.

To show that $B_{R}$ is summable $(C, n)$ to zero, we obtain (17) as in Lemma 8. Then from the fact that $A_{R}$ is $(C, n)$ summable to zero and from [3, Theorem $55]$, it is sufficient once again to show that (18) holds.

But by Parseval's formula, we obtain that the expression in (18) is equal to (19). Observing that for $u$ in $D_{n}\left(0, r_{0} / 2\right)-0$ and for $y$ in $E_{n}-D_{n}\left(0, r_{0}\right)$ there exists a constant $\bar{K}_{n}$ such that

$$
\left|J_{n+\mu-1}(R|y-u|)\right| \leq \bar{K}_{n}(R|y-u|)^{-1 / 2} \quad \text { for } R>1
$$

and that for such $u,|y-u|^{1 / 2-n}$ is in $L_{2}$ on $E_{n}-D_{n}\left(0, r_{0}\right)$, we conclude that 
(18) holds and consequently that $B_{R}$ is $(C, n)$ summable to zero, which proves the lemma.

5. Proof of Theorem 1. To prove Theorem 1, it is sufficient to show that for any $r_{0}>0, c(u)=0$ almost everywhere in $D_{n}\left(0, r_{0} / 2\right)$. Set

$$
F_{1}(x)=-\int_{D_{n}\left(0, r_{0}\right)} \frac{\left[e^{i(x, u)}-1-i(x, u)\right]}{|u|^{2}} c(u) d u
$$

Then, $F_{1}(x)$ is in $C^{(\infty)}$ on $E_{n}$ and

$$
\Delta F_{1}(x)=\int_{D_{n}\left(0, r_{0}\right)} e^{i(x, u)} c(u) d u .
$$

Set

$$
F_{2}(x)=-\int_{E_{n}-D_{n}\left(0, r_{0}\right)} e^{i(x, u)} \frac{c(u)}{|u|^{2}} d u
$$

which is by (iii) continuous in $E_{n}$. Then by Lemma 2 and (ii),

$$
\varlimsup_{r \rightarrow 0}\left|2 n \nabla_{1}\left(F_{2} ; x ; r\right) / r^{2}\right|<\infty
$$

in $E_{n}-Z$ and by Lemma 3 and $(\mathrm{i}), \Delta_{1} F_{2}(x)=-\Delta F_{1}(x)$ almost everywhere.

Set $F(x)=F_{1}(x)+F_{2}(x)$. Take any $x_{0}$ in $E_{n}$ and consider $D_{n}\left(x_{0}, r_{1}\right)$, $r_{1}>0$. From the definition of a closed set of vanishing capacity, we see that there is a closed bounded set of capacity zero $Z_{1}$ such that

$$
\varlimsup_{r \rightarrow 0}\left|2 n \nabla_{1}(F ; x ; r) / r^{2}\right| \leq\left|\Delta F_{1}(x)\right|+\varlimsup_{r \rightarrow 0}\left|2 n \nabla_{1}\left(F_{2} ; x ; r\right) / r^{2}\right|<\infty
$$

for $x$ in the domain $G=D_{n}\left(x_{0}, r_{1}\right)-Z_{1} D_{n}\left(x, r_{1}\right)$. Furthermore almost everywhere in $G, \Delta_{1} F(x)=\Delta F_{1}(x)+\Delta_{1} F_{2}(x)=0$. Consequently it follows from Lemma 7 , that $F(x)$ is harmonic in the domain $G=D_{n}\left(x_{0}, r_{1}\right)-Z_{1} D_{n}\left(x_{0}, r_{1}\right)$. But $F(x)$ is continuous in $D_{n}\left(x_{0}, r_{1}\right)$. Therefore by $\left[7, p_{0} 335\right], F(x)$ is harmonic in $D_{n}\left(x_{0}, r_{1}\right)$ and since $x_{0}$ is arbitrary, $F(x)$ is harmonic in $E_{n}$.

From the fact that $F(x)$ is harmonic in $E_{n}$, we now have that $F_{2}(x)=F(x)$ $-F_{1}(x)$ is in $C^{(\infty)}$ on $E_{n}$ and that $\Delta F_{2}(x)=-\Delta F_{1}(x)$ for all $x$. Also by [1, Theorem 1] we obtain that 


$$
(2 \pi)^{-n} \int_{E_{n}} e^{-i(x, u)} F_{2}(x) d x
$$

is spherically summable $(C, n)$ to zero for $u$ in $D_{n}\left(0, r_{0} / 2\right)-0$. Therefore by Lemma 8 for such $u$,

$$
\int_{E_{n}} e^{-i(x, u)}\left[-\Delta F_{2}(x)\right] d x
$$

is spherically summable $(C, n)$ to zero. But for almost all such $u$, we have that

$$
(2 \pi)^{-n} \int_{E_{n}} e^{-i(x, u)} \Delta F_{1}(x) d x
$$

is spherically summable $(C, n)$ to $c(u)$. Since $\Delta F_{1}(x)=-\Delta F_{2}(x)$, we conclude that for almost all $u$ in $D_{n}\left(0, r_{0} / 2\right), c(u)=0$, which proves the theorem.

6. Proof of Theorem 2. The proof is quite similar to that of Theorem 1. Once again it is sufficient to prove that for any $r_{0}>0, c(u)=0$ almost everywhere in $D_{n}\left(0, r_{0} / 2\right)$.

Set

$$
F_{1}(x)=-\int_{D_{n}\left(0, r_{0}\right)}\left[e^{i(x, u)}-1-i(x, u)\right] \frac{c(u)}{|u|^{2}} d u
$$

and

$$
F_{2}(x)=-\lim _{R \rightarrow \infty} \int_{D_{n}\left(0, r_{R}\right)-D_{n}\left(0, r_{0}\right)} e^{i(x, u)} \frac{c(u)}{|u|^{2}} d u
$$

By (iii), $F_{2}(x)$ is continuous. Then in a manner exactly analogous to the proof of Theorem 1 except that Lemmas 4, 5, and 6 are used instead of 2, 3, and 7, we obtain that $F_{2}(x)$ is in $C^{(\infty)}$ and that $\Delta F_{2}(x)=-\Delta F_{1}(x)$. By Lemma 9 and [3, Theorem 55], we obtain that $\int_{E_{n}} e^{-i(x, u)}\left[-\Delta F_{2}(x)\right] d x$ is spherically summable $(C, n)$ to zero for $u$ in $D_{n}\left(0, r_{0} / 2\right)-0$. But by [1, Theorem 1] for almost all such $u$, we have that

$$
(2 \pi)^{-n} \int_{E_{n}} e^{-i(x, u)} \Delta F_{1}(x)
$$

is spherically summable $(C, n)$ to $c(u)$. Since $-\Delta F_{2}(x)=\Delta F_{1}(x)$, we conclude that $c(u)=0$ almost everywhere in $D_{n}\left(0, r_{0} / 2\right)$ and the theorem is proved. 
7. Proof of Theorem 3. Let $F_{1}(x)$ be as in Theorem 2 with $n$ replaced by 2 , and let

$$
F_{2}(x)=-\lim _{R \rightarrow \infty} \int_{D_{2}(0, R)-D_{2}\left(0, r_{0}\right)} \frac{c(u)}{|u|^{2}} e^{i(x, u)}
$$

where $r_{0}>0$. This limit exists for $x$ in $Z$ by (ii) and for $x$ not in $Z$ by (i), (iii), and Lemma 1. Furthermore by (ii) $F_{2}(x)$ is assumed continuous in $E_{2}-W$. It is clear from the proof of Theorem 2 that to prove this theorem we need only show that $F_{2}(x)$ is continuous in $E_{2}$ or what is the same thing that $F(x)=$ $F_{1}(x)+F_{2}(x)$ is continuous in $E_{2}$.

By (ii) $F(x)$ is continuous in $E_{2}-\mathbb{W}$, and by Lemmas $5, \Delta_{2} F(x)=0$ in $E_{2}-Z$. Let $D_{2}\left(x_{0}, r_{1}\right)$ be any disc which has a null intersection with $\mathbb{W}$. Then as in the proof to Theorem $1, F(x)$ is harmonic in this disc and consequently in $E_{2}-\mathbb{W}$. We also observe that now $\Delta_{2} F(x)=0$ in the whole plane and furthermore that $F(x)$ is in $L_{2}$ on any bounded domain.

Let $W_{1}$ be the set of discontinuity points of $F(x)$ and let $x_{0}$ be an isolated point of $W_{1}$. Then there is a closed disc $\bar{D}_{2}\left(x_{0}, r_{2}\right)$ whose intersection with $\mathbb{W}_{1}^{\prime}$ is $x_{0}$. Then by the above discussion we have that $F(x)$ is in $L_{2}$ on $D_{2}\left(x_{0}, r_{2}\right)$, harmonic in $D_{2}\left(x_{0}, r_{2}\right)-x_{0}$, and satisfies the further condition that $\Delta_{2} F\left(x_{0}\right)=0$. Consequently by [12, Lemma 4$], F(x)$ is then harmonic in the whole disc and, a fortiori, continuous at $x_{0}$.

Therefore $W_{1}$, has no isolated points and $\bar{W}_{1}$ is a perfect set. But $\bar{W}_{1} \subset W$ is at most denumerable, and by $\left[10\right.$, p. 55], $\bar{W}_{1}$ is then the empty set. Thus $F(x)$ is continuous in the whole plane, and, as mentioned above, the proof of this theorem is reduced to that of Theorem 2 .

8. Appendix. In closing we point out that the assumption $W$ and $Z$ have a null intersection in Theorem 3 is a necessary one. For consider the double trigonometric integral $\int_{E_{2}} c(u) e^{i(x, u)} d u$ with $c(u)=1$. (iii) and (iv) of Theorem 3 are clearly satisfied. Observing that the spherical mean of rank $R$, $\sigma_{R}^{(1)}(x)$, with $x \neq 0$ is given by

$$
\sigma_{R}^{(1)}(x)=4 \pi J_{2}(|x| R)|x|^{-2}=O\left(R^{-1 / 2}\right)
$$

we see that (i) is satisfied with $Z$ equal to the origin. Furthermore, we observe that for $x \neq 0$

$$
\lim _{R \rightarrow \infty} \int_{D_{2}(0, R)-D_{2}(0,1)}|u|^{-2} e^{i(x, u)} d u=2 \pi \int_{|x|}^{\infty} J_{0}(r) r^{-1} d r .
$$


Consequently (ii) is satisfied with $W$ consisting of the origin. But $W$ and $Z$ do not have a null intersection, and the conclusion of Theorem 3 does not hold.

\section{REFERENCES}

1. S. Bochner, Summation of multiple Fourier series by spherical means, Trans. Amer. Math. Soc. 40 (1936), 175-207.

2. - Theta relations with spherical harmonics, Proc. Nat. Acad. Sci., 37 (1951), $804-808$.

3. S. Bochner and K. Chandrasekharan, Fourier transforms, Princeton, 1949.

4. M. Brelot, Sur la structure des ensembles de capacité nulle, C. R. Acad. Sci. Paris 192 (1931), 206-208.

5. M. T. Cheng, Uniqueness of multiple trigonometric series, Annals of Math. 52 (1950), $403-416$. 1937.

6. R. Courant and D. Hilbert, Methoden der mathematischen Physik, vol. 2 Berlin,

7. O. D. Kellogg, Foundation of potential theory, Berlin, 1929.

8. T. Rado, Subharmonic functions, Ergebnisse der Mathematik, vol. 5, no. 1, Berlin, 1937.

9. W. Rudin, Integral representations of continuous functions, Trans. Amer. Math. Soc. 68 (1950), $278-286$.

10. S. Saks, Theory of the integral, 2d. ed., Warsaw, 1937.

11. V.L. Shapiro, An extension of results in the uniqueness theory of double trigonometric series, Duke Math. J. 20 (1953), 359-366.

12. - A note on the uniqueness of double trigonometric series, Proc. Amer. Math. Soc. 4 (1953), $692-695$.

13. - Summability and uniqueness of double trigonometric integrals, Trans. Amer. Math. Soc., 77 (1954), 322 - 339.

14. G. N. Watson, A treatise on the theory of Bessel functions, Cambridge, 1944.

15. A. Zygmund, Trigonometrical series, Warsaw, 1935.

Rutgers University, New BrUNSWICK, NEW JERSEY AND

The Institute for Advanced Study, Princeton, New Jersey 